\title{
Estereotipos de género, maternidad y empleo: un análisis psicosociológico
}

\author{
Gender Stereotypes, Maternity and Employment: \\ A Psychosociological Analysis
}

\author{
Alicia Garrido-Luque ${ }^{1} \bowtie$, José Luis Álvaro-Estramiana ${ }^{2}$, \\ Ana Raquel Rosas-Torres ${ }^{3}$
}

\author{
${ }^{1}$ Universidad Complutense de Madrid, España \\ ${ }^{2}$ Universidad Complutense de Madrid, España \\ ${ }^{3}$ Universidad Federal de Paraiba, Brasil
}

$\triangle$ Av. Séneca, 2, 28040 Madrid, España. Correo electrónico: algarrid@cps.ucm.es

Recibido: 8 de marzo del $2017 \quad$ Aprobado: 27 de noviembre del 2017 Disponible en línea: 1 de abril de 2018

Cómo citar este artículo: Garrido-Luque, A., Álvaro-Estramiana, J. L., y Rosas-Torres, A. R. (2018). Estereotipos de género, maternidad y empleo: un análisis psicológico. Pensando Psicología, 14(23). doi: https://doi.org/10.16925/pe.v14i23.2261

\section{Resumen}

Introducción: en las últimas décadas se ha producido un cambio sustancial en la posición de las mujeres en el mercado laboral, aunque no puede hablarse todavía de un reparto equitativo del trabajo entre hombres y mujeres. Por una parte, existen aún claros indicios de discriminación laboral de las mujeres, y por otro, la participación de los varones en el trabajo doméstico no ha crecido al ritmo al que lo ha hecho la de la mujer en el trabajo remunerado.

Objetivo: reflexionar sobre las bases ideológicas en las que se sustenta esta desigualdad mediante el análisis del peso que los estereotipos de género tradicionales, y específicamente aquellos que se construyen alrededor de la maternidad, tienen en la carrera laboral de las mujeres jóvenes.

Metodología: los datos proceden de un estudio cualitativo en el que se entrevistó a una muestra de jóvenes con diferentes posiciones dentro del mercado de trabajo.

Resultados: en general, los discursos sobre la maternidad y la crianza se caracterizan por un marcado esencialismo biológico y psicológico que atribuye a las mujeres una mayor capacidad natural para el cuidado de los hijos.

Conclusiones: Ios estereotipos de género que se construyen alrededor de la maternidad son uno de los principales factores ideológicos que mantienen la discriminación de las mujeres dentro del mercado laboral.

Palabras clave: estereotipos de género, exclusión laboral, maternidad, paternidad, tiempo de trabajo. 


\title{
Gender Stereotypes, Maternity and Employment: A Psychosociological Analysis
}

\begin{abstract}
Introduction: In the last decades there has been a substantial change in the position of women in the labor market, although it cannot yet be said that there is an equal share of jobs between men and women. On the one hand, there are still clear indications of employment discrimination against women and, on the other hand, the participation of men in domestic work has not grown at the same rate as that of women in paid work.

Objective: To reflect on the ideological bases supporting this inequality by analyzing the weight that traditional gender stereotypes, especially those built around motherhood, have on the career of young women.

Method: Data come from a qualitative study in which a sample of young people with different positions within the labor market was interviewed.

Results: Generally, the discourses on maternity and upbringing are characterized by a marked biological and psychological essentialism that attributes a greater natural capacity for the care of children to women.
\end{abstract}

Conclusions: Gender stereotypes that are built around motherhood are one of the main ideological factors that maintain discrimination against women in the labor market.

Keywords: gender stereotypes, labor exclusion, motherhood, fatherhood, work time.

\section{Estereótipos de gênero, maternidade e emprego: uma análise psicosociológica}

\section{Resumo}

Introdução: nas últimas décadas ocorreu uma mudança substancial na posição das mulheres no mercado de trabalho, mesmo que ainda não seja possível falar em uma divisão igualitária do trabalho entre homens e mulheres. Por um lado, ainda existem claros indícios de discriminação laboral com as mulheres e, por outro lado, a participação dos homens no trabalho doméstico não cresceu no mesmo ritmo da participação da mulher no trabalho remunerado.

Objetivo: refletir sobre as bases ideológicas nas quais essa desigualdade é sustentada através da análise do peso que os estereótipos de gênero tradicionais, e especificamente aqueles que são construídos ao redor da maternidade, têm na carreira profissional das mulheres jovens.

Metodologia: os dados procedem de um estudo qualitativo no qual entrevistou-se uma amostra de jovens com diferentes posições dentro do mercado de trabalho.

Resultados: em geral, os discursos sobre a maternidade e a criação são caracterizados por um essencialismo biológico e psicológico marcado que atribui às mulheres uma maior capacidade natural para 0 cuidado dos filhos.

Conclusões: os estereótipos de gênero que são construídos ao redor da maternidade são um dos principais fatores ideológicos que mantêm a discriminação com as mulheres dentro do mercado de trabalho.

Palavras-chave: estereótipos de gênero, exclusão laboral, maternidade, paternidade, tempo de trabalho. 


\section{Introducción}

La división tradicional de los roles de género, la cual adscribe al varón el papel de sustentador económico de la familia y a la mujer el cuidado físico y afectivo de esta, dio lugar históricamente y de manera progresiva a la forma más explícita de exclusión de las mujeres del mundo laboral: la negación misma de la posibilidad de participar en él. Aunque todavía no se puede hablar de una completa equiparación de la posición de varones y mujeres en el mercado de trabajo, podría afirmarse que esta forma extrema de exclusión se hizo gradualmente menos visible a lo largo del siglo $\mathrm{xx}$, aunque hasta bien entrado este la permanencia de las mujeres en el mundo laboral estaba condicionada a la no existencia de responsabilidades familiares. El trabajo de las mujeres era socialmente aceptado, siempre y cuando no entrara en colisión con la división de roles de la sociedad patriarcal. En el caso de España, por ejemplo, en la década de 1970, apenas un tercio de las mujeres con edades comprendidas entre los 25 y los 54 años permanecía en el mercado laboral. Este dato, junto con la enorme distancia que separaba a las tasas de ocupación masculinas y femeninas (96\% frente a 29\%), nos habla de una sociedad con una fuerte división del trabajo entre ambos géneros. La situación es radicalmente diferente en la actualidad. En el primer trimestre del 2017, alrededor del $80 \%$ de las mujeres de entre 25 y 54 años formaba parte de la población activa, y más del $60 \%$ tenía un empleo, lo que evidencia un cambio sustancial en el modelo de familia, en el sentido de que el varón ya no es el sustentador exclusivo de esta y la mujer ya no abandona el mercado laboral después del nacimiento de los hijos y las hijas (INE, 1976; 2017).

Sin embargo, a pesar de los indudables progresos que se han producido en la dirección de una mayor integración de las mujeres en el mercado laboral, no podemos hablar todavía de plena igualdad en el reparto del trabajo. Las mujeres tienen mayor nivel de precariedad laboral, menores salarios y más dificultades para ascender en la escala ocupacional y ocupar puestos directivos y de responsabilidad (Carrasquer, 2009; Pastor, 2009; Torns y Recio, 2012). Además, la formación de una familia y, sobre todo, la llegada de los hijos y las hijas sigue afectando de forma diferente la trayectoria laboral de hombres y mujeres. Algunos estudios recientes demuestran que la especialización de roles de género se intensifica tras el nacimiento del primer hijo. Mientras que el tránsito a la paternidad coincide con la consolidación del rol profesional del hombre, la maternidad se asocia con una intensificación de las actividades en el ámbito doméstico, lo cual puede tener efectos en el desarrollo de la carrera profesional de la mujer (Flaquer y Escobedo, 2014).

El objetivo de este artículo es reflexionar sobre algunas de las bases ideológicas en las que se sustenta esta persistente desigualdad. Más específicamente, nuestro objetivo es describir las creencias que las personas jóvenes tienen sobre el papel de hombres y mujeres en la crianza de los hijos y las hijas, y analizar la influencia que dichas creencias pueden tener en la posición en el mercado laboral.

\section{Estereotipos de género y división del trabajo}

Una de las funciones que cumplen los estereotipos es justificar la discriminación y la exclusión social que sufren algunos grupos. Desde la psicología social, autores como Allport (1954) o Tajfel (1981) han reflexionado sobre la función de racionalización que los estereotipos cumplen en el contexto de las relaciones intergrupales. En el caso de los estereotipos de género, las creencias sobre la diferente caracterización psicológica de hombres y mujeres han servido históricamente para racionalizar la división del trabajo, y legitimar la exclusión de estas del mercado laboral y de la esfera pública en general. Aunque la reflexión sobre los vínculos entre el trabajo doméstico y la psicología femenina es muy antigua (Durán, 2006), fue durante las primeras décadas del siglo $\mathrm{xx}$ cuando la contraposición entre los rasgos psicológicos femeninos y masculinos se convirtió en objeto de fuertes debates, que han sido interpretados como una reacción ante los avances del movimiento feminista y la incipiente aparición de las mujeres en diferentes ámbitos de la esfera pública (Osborne, 1987). Comenzaron a proliferar durante esta época las dicotomías que establecían una profunda diferenciación entre los rasgos psicológicos del varón, más acordes con la dinámica del espacio público, y los de la mujer, que la situaban de manera natural en el ámbito del hogar y de las relaciones sociales. Un ejemplo temprano de este tipo de dicotomías en la sociología de la época lo encontramos en Simmel, quien dedicó varios ensayos a reflexionar sobre la diferenciación psicológica y social entre hombres y mujeres (Simmel, 1938). El autor estableció una clara contraposición entre los rasgos masculinos - vinculados a lo que denominó "el mundo objetivo", relacionado con la 
actividad, la creatividad o la proyección exterior-, y los rasgos femeninos - asociados a un mundo subjetivo, más relacionado con la afectividad, la reproducción y la vida familiar-.

La tendencia a utilizar este tipo de dicotomías para diferenciar psicológicamente a hombres y mujeres se encuentra profundamente arraigada en la cultura popular. Sin entrar aquí en la cuestión de si estas diferencias existen realmente o no, lo cual sería objeto de la psicología diferencial, lo que la psicología social ha demostrado es que la gente cree que existen y utiliza dicotomías muy similares a la de Simmel para describir a hombres y mujeres. Las primeras investigaciones en las que se analizó el contenido de los estereotipos de género (Broverman, Vogel, Broverman, Clarkson y Rosenkrantz, 1972; Rosenkrantz, Vogel, Bee, Broverman y Broverman, 1968) establecieron una distinción entre los rasgos relacionados con la competencia y los asociados a la expresividad emocional, dicotomía que es conceptualmente similar a la que estudios posteriores utilizaron para distinguir entre instrumentalidad y expresividad (Spence, Helmrecih y Stapp, 1975), o entre agencia y comunalidad (Deaux y Lewis, 1984; Eagly y Steffen, 1984; Williams y Best, 1982). En todas estas dicotomías los hombres se perciben como competentes, asertivos, independientes y orientados hacia el poder y el logro, mientras que las mujeres se perciben como afectivas, dependientes, sociables, poco preocupadas por ellas mismas y orientadas hacia los demás.

Este tipo de creencias no llevan implícita necesariamente una peor valoración de las mujeres. De hecho, pese a que los primeros estudios sobre estereotipos de género sí sugerían que a ellas las percibían de forma más negativa con relación a los varones, el desarrollo posterior de la investigación llevó a identificar formas más complejas de sexismo en las que la percepción estereotipada de las mujeres no se sustenta necesariamente en una percepción negativa de ellas. Un ejemplo de esto lo encontramos en el concepto de sexismo ambivalente propuesto por Glick y Fiske (1996). Estos autores identificaron dos dimensiones independientes dentro del sexismo: el sexismo hostil, que implica una evaluación negativa de las características femeninas, y el sexismo benévolo, en el que la percepción estereotipada de las mujeres va acompañada de la expresión de sentimientos positivos hacia ellas. Esta percepción más positiva de las mujeres no es incompatible con su discriminación en el mercado laboral. Tal y como lo señalan Fiske (1993), y Fiske Cuddy, Glick y Xu (2002), el prejuicio no se basa en una única dimensión de antipatía o simpatía hacia diferentes grupos, sino que estos se evalúan en dos dimensiones independientes de agrado y respeto, asociadas respectivamente al afecto y a la competencia que se les percibe. Existen determinados grupos que gozan de un alto grado de respeto porque se perciben como competentes, pero al mismo tiempo generan antipatía debido a la frialdad y la falta de afectividad que se les atribuye. Otros grupos, sin embargo, despiertan sentimientos de simpatía porque se les asignan rasgos relacionados con la esfera de la afectividad, pero se consideran poco competentes y, por tanto, no generan respeto. En el primer caso estaríamos ante formas hostiles de estereotipia y discriminación, mientras que en el segundo supuesto estaríamos ante estereotipos de carácter paternalista, asociados a formas benevolentes de discriminación. El hecho de que en algunos estudios se observen actitudes más positivas hacia las mujeres se debe, fundamentalmente, a la alta valoración que estas consiguen en rasgos relacionados con la afectividad y la empatía. Esto da lugar a lo que algunos autores han denominado como el efecto "las mujeres son maravillosas" (Eagly y Mladinic, 1994), una especie de sexismo benevolente que evalúa de forma muy favorable a las mujeres pero no evita su discriminación, ya que no son estos precisamente los rasgos más valorados en el mercado laboral. Lo que da lugar a la discriminación no es, por tanto, la actitud más o menos positiva hacia las mujeres, sino la falta de ajuste entre los rasgos propios del estereotipo femenino y los perfiles psicológicos que se valoran en el mercado laboral (Heilman, 2012; Heilman y Eagly, 2008), algo especialmente evidente en las ocupaciones consideradas tradicionalmente como masculinas, así como en los puestos de dirección y responsabilidad. Esta falta de ajuste no afecta únicamente a las mujeres; también los varones que intentan acceder a empleos típicamente femeninos se ven discriminados por la percepción estereotipada de los rasgos de la masculinidad.

Según la teoría de los roles sociales (Eagly, 1987), las diferencias entre hombres y mujeres observadas en la investigación psicológica tienen su origen en la estructura social, la cual establece una diferenciación entre los roles de género. La necesidad de ajustarse a las expectativas de los roles que les han sido asignados hace que hombres y mujeres adquieran y desarrollen características psicológicas y comportamientos diferentes. Son estas diferencias en los roles sociales y laborales que desempeñan hombres y mujeres 
las que dan lugar a la formación y el mantenimiento de los estereotipos de género (Eagly y Steffen, 1984). En este sentido, es previsible que el cambio que se ha producido en los roles laborales de las mujeres haya tenido algún efecto en el contenido de los estereotipos. En algunos estudios recientes (Castillo-Mayén y Montes-Berges, 2014) se ha observado que, si bien hubo un cambio significativo en los estereotipos de género - especialmente en los femeninos-, algunos de los estereotipos tradicionales siguen vigentes. Rasgos como egoístas, fuertes o insensibles aún se asignan con más frecuencia a los hombres, mientras que adjetivos como dulce, emocional o comprensiva se perciben como más típicos de las mujeres (2014). La diferenciación psicológica entre hombres y mujeres, así como la atribución a estas de este tipo de rasgos favorables relacionados con la afectividad y la empatía, se utiliza a veces para legitimar la división de roles dentro del hogar y las diferencias de posición en el mercado de trabajo (Cundiff y Vescio, 2016; Hoffman y Hurst, 1990; Jackman, 1994; Jost y Banaji, 1994; Jost y Kay, 2005; Koening y Eagly, 2014).

\section{Estereotipos sobre la maternidad}

Si los estereotipos de género contribuyen en general a mantener la diferenciación de hombres y mujeres dentro del mercado laboral, los que se construyen alrededor de la maternidad se convierten en un poderoso mecanismo de exclusión y discriminación. La presencia en el hogar de hijos e hijas menores condiciona fuertemente la percepción que se tiene del empleo femenino. Algunos de los datos del último estudio sobre familia y género del Centro de Investigaciones Sociológicas (CIS, 2004) sugieren que la maternidad y la paternidad se construyen aún en gran medida sobre la base de la diferenciación tradicional de los roles de la madre y el padre. La mayoría de las personas encuestadas (52\% de los hombres y $61 \%$ de las mujeres) cree que en el caso de que haya hijos e hijas en edad preescolar, la madre debe reducir su jornada y trabajar a tiempo parcial, y un porcentaje nada despreciable ( $28 \%$ de los varones y $20 \%$ de las mujeres) considera que la madre no debería trabajar en este caso. Tan solo una minoría (12\% de hombres y $11 \%$ de mujeres) piensa que la madre debe mantener su empleo a jornada completa. La situación se invierte en el caso del padre. Frente a una clara mayoría ( $78 \%$ de los varones y $72 \%$ de las mujeres) que considera que este debe seguir trabajando a jornada completa, una minoría (14\% de los varones y
$20 \%$ de las mujeres) cree que ambos miembros de la pareja deberían trabajar a tiempo parcial, de modo que no es apreciable el porcentaje de personas que cree que el padre no debe trabajar cuando hay hijos e hijas en edad preescolar. La diferente percepción del empleo masculino y femenino se encuentra sustentada en creencias fuertemente arraigadas sobre el papel diferente que desempeñan las figuras materna y paterna en la crianza de los hijos. En el mismo estudio del cIs sobre familia y género (CIs, 2004), alrededor de la mitad de los encuestados (54\% de los varones y $47 \%$ de las mujeres) cree que a un niño en edad preescolar le perjudica que su madre trabaje, y el $57 \%$ de los varones y el $61 \%$ de las mujeres creen que la vida familiar se resiente cuando la mujer tiene un empleo de jornada completa. El hecho de que en el estudio no se incluya ninguna pregunta sobre la presencia del padre en el hogar o el vínculo entre el padre y los hijos es de por sí indicador de la diferente percepción social de la maternidad y la paternidad.

Las investigaciones en las que se ha analizado la experiencia de la maternidad muestran que el estereotipo de la buena madre todavía se construye alrededor de creencias universalistas y esencialistas sobre la naturaleza femenina (Park, Banchefsky y Reynolds, 2015). La idea según la cual la maternidad es la principal vía de realización de la mujer, y que ella está capacitada de forma natural e instintiva para el cuidado de los hijos, se encuentra fuertemente arraigada no solo en la cultura popular, sino también en el discurso médico y en los medios de comunicación (Bobel, 2002; Hays, 1996; Oakley, 1979; Sullivan, 2015; Wall, 2013). Se cree que, como expresión del instinto maternal, la mujer desarrolla una serie de rasgos psicológicos vinculados a la expresividad emocional y la afectividad que la predisponen y capacitan para el cuidado de los hijos, así como la diferencian psicológicamente del varón, quien no posee de manera natural estas capacidades. Esta percepción diferencial de la maternidad y la paternidad que ha sido observada en algunos estudios recientes (Bravo, FiguerasMaz y Gómez-Puertas, 2014; Rodríguez-Menéndez y Fernández-García, 2010; Rodríguez, Peña y Torío, 2009), contribuye a justificar la posición diferente de hombres y mujeres en el mercado laboral.

Aunque el carácter normativo de los estereotipos sobre la maternidad no es actualmente tan fuerte como lo fue en el pasado, las expectativas asociadas al rol maternal están todavía hoy condicionadas en gran medida por el modelo tradicional de la maternidad, 
basado en la entrega y la presencia permanente de la madre. La identificación con este modelo es más fuerte en las mujeres más tradicionales en cuanto a sus actitudes hacia el rol de género (Paterna, Martínez, Rosa y Yago, 2001).

Por otra parte, la maternidad condiciona en gran medida la percepción social de las mujeres. Cuando la mujer es percibida como madre, se exacerba su feminidad y se hacen más sobresalientes los rasgos típicos del estereotipo femenino, más relacionados con el cuidado y la emotividad, y más alejados de los perfiles psicológicos que se valoran en mayor medida en el mercado laboral. Algunas investigaciones muestran que las mujeres que han tenido hijos recientemente se evalúan como menos competentes y menos comprometidas con el trabajo frente a las mujeres que no los tienen y a los hombres, independientemente de que estos sean o no padres (Crosby, Williams y Biernat, 2004; Cuddy, Fiske y Glick, 2004; Heilman y Okimoto, 2008; Ridgeway y Correll, 2004).

A las evaluaciones negativas que se derivan de la exacerbación de los rasgos del estereotipo femenino durante la maternidad, hemos de añadir las desventajas que se derivan de la falta de equidad que aún existe en el reparto del trabajo doméstico. A pesar de que en el plano de los discursos se expresa hoy en día una clara preferencia por un modelo de familia igualitario, en el que hombre y mujer participen del mismo modo en los trabajos remunerado y doméstico, la participación de los varones en este ámbito no ha aumentado de forma proporcional a como lo ha hecho la participación de las mujeres en el mercado laboral (Domínguez-Folgueras, 2010; Durán, 2000; 2005; Durán y Rogero, 2005; García Román, 2012). El hecho de que la definición social de la maternidad descanse aún en estereotipos tradicionales sobre la diferenciación psicológica entre hombres y mujeres hace que la presencia de estas en el hogar se perciba todavía como imprescindible cuando hay hijos. La idea de que la maternidad va a limitar el tiempo que las mujeres pueden dedicar a su trabajo, basada en el hecho de que son ellas las principales responsables del cuidado de los hijos, da lugar a una percepción negativa de la implicación de la mujer con su carrera laboral.

\section{Método}

Los datos que se presentan en este artículo proceden de una investigación sobre la percepción que las personas jóvenes tienen del proceso de inserción e integración en el mercado laboral. Dicha investigación fue financiada por el Instituto de la Mujer (Ministerio de Asuntos Sociales e Igualdad), y algunos de sus resultados han sido publicados en el informe de investigación correspondiente. La investigación se llevó a cabo en Madrid (España). Con respecto al método, se adoptó un enfoque cualitativo, utilizándose la entrevista semiestructurada como principal técnica de recogida de datos. Se realizaron 36 entrevistas a 24 mujeres y 12 varones jóvenes con edades entre los 20 y los 35 años. Las variables que se utilizaron como criterios a la hora de seleccionar a la muestra fueron la situación laboral, la edad y el nivel de estudios. En cuanto a este último se distinguió entre estudios primarios, educación secundaria, formación profesional y estudios universitarios; se realizaron nueve entrevistas (seis mujeres y tres varones) para cada nivel. La sobrerrepresentación de mujeres en la muestra estuvo motivada por el interés en analizar la forma en que la percepción de la maternidad y los planes de formar una familia influyen en la experiencia laboral. De los y las jóvenes entrevistadas, 25 (nueve varones y 16 mujeres) tenían un empleo, y 11 (tres varones y ocho mujeres) estaban sin empleo, es decir, no tenían un empleo y se encontraban buscando trabajo en el momento en que se realizaron las entrevistas. En cuanto al tipo de trabajos desempeñados, tres de los entrevistados (todos ellos varones) eran trabajadores autónomos, mientras que el resto trabajaba por cuenta ajena en empleos de diferente nivel de cualificación profesional. De los trabajadores por cuenta ajena, un total de 13 (10 mujeres y tres varones) tenían empleos poco cualificados en hostelería y comercio, y 12 (nueve mujeres y tres varones) tenían empleos cualificados en empresas del sector servicios y de la banca.

De de los y las 36 jóvenes entrevistadas, un total de seis (cuatro mujeres y dos varones) tenían hijos, todos ellos menores de tres años, y 10 (ocho mujeres y dos varones) planificaban tenerlos.

Las entrevistas fueron semiestructuradas, y en ellas se recogió información sobre la trayectoria laboral, la situación laboral actual, los significados atribuidos al trabajo remunerado, la identificación con el trabajo doméstico, las expectativas relacionadas con la maternidad y la paternidad, y la percepción de las dificultades de conciliación entre familia y empleo. A continuación, se describen los resultados del análisis de contenido. 


\section{Resultados}

\section{Estereotipos sobre la maternidad y la paternidad}

La información obtenida en este estudio nos lleva a destacar, en primer lugar, la alta centralidad que el empleo tiene para las mujeres jóvenes, algo en lo que estas no se diferencian significativamente de los varones de la misma edad y del mismo nivel educativo. Esta alta centralidad coexiste, no obstante, con una imagen marcadamente estereotipada de la maternidad y con una fuerte identificación con la definición tradicional del rol maternal.

En general, los discursos sobre la maternidad y la crianza se caracterizan por un marcado esencialismo biológico y psicológico que atribuye a las mujeres una mayor capacidad natural para el cuidado de los hijos. Se establece una continuidad entre los procesos biológicos que rodean al embarazo y el parto, y los procesos sociales relacionados con la crianza. En virtud de su fisiología y de la unión biológica que hubo durante el embarazo, se considera que la mujer tiene una capacidad instintiva para detectar las necesidades del hijo, una especie de sexto sentido que la mantiene en un estado de alerta y preocupación permanentes:

Estás permanentemente en alerta, es que antes de que llore ya sé que va a llorar, me despierto sin necesidad de que llore. Es como si estuviera programado. ( $\mathrm{Mu}-$ jer, 30 años, estudios superiores)

[...]

Si es que el instinto te lo dice, te dice lo que tienes que hacer. Al principio tienes mucho miedo, pero después te das cuenta de que lo sabes hacer por instinto. (Mujer, 28 años, estudios medios)

Es usual que esta imagen se acompañe de una imagen muy estereotipada del papel que los varones desempeñan como padres. A la imagen de la madre intuitiva y siempre vigilante se contrapone con frecuencia la del padre ignorante y despreocupado. De los hombres se resalta en muchas ocasiones su incapacidad para detectar las necesidades de los hijos y las hijas, su tranquilidad e imperturbabilidad, así como su torpeza natural para realizar algunas de las tareas domésticas relacionadas con el cuidado de los hijos: "Es que él no lo oye. Yo me despierto antes de que empiece a llorar, como que sé cuándo va a empezar a llorar y ya estoy yo atenta. Y lo miro, y durmiendo tranquilamente" (mujer, 30 años, estudios superiores).

Le cambió el pañal y se lo puso al revés, y la pobre niña llorando porque estaba incómoda, y sin saber que le pasa, y es que le había puesto el pañal al revés y le molestaba. Si es que no saben, y para que lo haga mal, prefiero hacerlo yo desde el principio, y encima acabo antes. (Mujer, 30 años, estudios medios)

Esta imagen estereotipada de la menor capacidad del varón para desenvolverse en la esfera emocional y doméstica termina por servir como justificación de la división tradicional de roles y legitima la permanencia de la mujer en el ámbito doméstico. Dado que el hombre no está psicológicamente capacitado para el cuidado físico y emocional de los hijos, y que la mujer posee estas capacidades de forma natural, es la presencia de la madre en el hogar - y no la del padre- la que se vuelve imprescindible para el adecuado desarrollo de aquéllos. Esta diferenciación psicológica, junto con los procesos biológicos que rodean al embarazo y al parto (incluida la lactancia materna) se utilizan como argumentos para justificar el hecho de que sea la madre la que abandone temporalmente su carrera laboral para cuidar de los hijos y las hijas. Incluso en aquellos casos en los que se contempla la posibilidad de que los varones disfruten de un permiso laboral por paternidad, su presencia en el hogar no se entiende como sustitutiva de la de la madre, sino más bien complementaria.

No sería justo que el hombre tenga la baja. Es ella la que ha tenido que sufrir el embarazo, así que es justo que sea ella la que pueda dejar de trabajar. Además, está todo el tema del pecho, que es la mujer la que lo tiene que hacer, es lógico que sea ella la que deje de trabajar. El hombre puede ayudar, pero por lógica no puede criar al hijo. (Hombre, 27 años, estudios medios)

[...]

Yo la maternidad quiero disfrutarla, entonces no me importará dejar unos meses el trabajo. Y está el tema del pecho, que es muy importante, y si eres tú la que da el pecho, no va irse de baja él, ¿no?. Tendrás que ser tú. (Mujer, 30 años, estudios superiores)

[...]

La baja maternal siempre en la madre, además por el tema del pecho y demás, que siempre es lo más im- 
portante. Lo ideal sería los dos juntos, eso es lo ideal. No compartir la baja de maternidad entre los dos, no cederla, sino tener los dos el mismo tiempo, que sería lo suyo. (Mujer, 26 años, estudios medios)

En las entrevistas realizadas con varones, hemos encontrado dos tipos de discurso sobre la experiencia de la paternidad. En uno de ellos, más frecuente en varones con menor nivel educativo, la paternidad tiene todavía hoy como referente el papel tradicional del varón como sustentador económico de la familia. En estos casos no suelen contraponerse trabajo y familia, y en general, la conciliación entre ambas esferas no aparece como un motivo de preocupación tan frecuente como se observa en el caso de las mujeres. El discurso se centra en la capacidad económica de mantener a los hijos y las hijas, más que en la posibilidad de tener tiempo para brindarles protección, lo que indica que muchos jóvenes siguen identificándose con el rol tradicional de sustentadores económicos de la familia, aunque matizado por la presencia de las mujeres en el mercado laboral. El hecho de que la mujer tenga un empleo no solo se acepta, sino que se considera absolutamente necesario para el mantenimiento económico de la familia. Sin embargo, no se asumen completamente las consecuencias que se derivan del discurso de la igualdad. El hecho de verse descargados de parte de la responsabilidad del sustento económico no les hace percibir el trabajo doméstico como una obligación. La identificación con un modelo de familia en el que ambos miembros de la pareja trabajen no implica, en estos casos, una mayor disposición a participar en el trabajo doméstico, el cual sigue percibiéndose de manera natural como una responsabilidad de las mujeres.

Sí, me gustaría tenerlo ahora, con nuestra edad, pero tendría que tener una casa, meterme en una casa, tendría que meterme luego en mantener el hijo que barato no es y... sí me gustaría. No sé, a mí los críos me encantan, pero no, no puedo. Ahora mismo no podría. (Hombre, 28 años, FPI)

$[\ldots]$

Pues actualmente la economía, el precio de los pisos, el precio de la vida en general. Ya hoy en día tienen que estar la pareja los dos trabajando. Eso de mantener uno la familia, eso ya es muy difícil. (....) La mujer lo tiene muy difícil. Tú ten en cuenta que en una casa es la mujer la que realmente trabaja. El hombre, por mucho que digamos, no es tanta la responsabilidad como la de la mujer. La mujer siempre está limpiando, fregando, esto, lo otro... Es la que más o menos dirige la casa. El hombre es el que trae el sueldo y la mujer, actualmente, por suerte también, pero la que lleva sobre todo el peso de la casa es la mujer. El hombre como que se desentiende un poquito; llevamos, pero no tanto. (Hombre, 26 años, estudios medios)

Junto con esta visión más tradicional, se observa un segundo tipo de discurso más igualitario. Se trata de jóvenes varones que han asumido de forma más consecuente la nueva posición de las mujeres dentro del mercado laboral y perciben claramente el efecto que esto ha de tener en su participación en el trabajo doméstico. Este discurso lo hemos observado con más frecuencia en jóvenes con un nivel educativo alto y cuya pareja está implicada en el desarrollo de una carrera profesional.

Yo estaría más contento haciendo tu trabajo tranquilamente (...) y si se normalizara el tema de las horas esas de por la tarde [sic] pues sería algo muy positivo para mí, vamos (...) tendría más libertad de por las tardes estar con la familia o de poder cuidar a los niños. Mi mujer también trabaja por la mañana y por la tarde y entonces a la hora de tener a un hijo pues estamos viendo que sería un poco complicado el compaginarlo, las dos cosas. Pues sería muy triste tener a un niño en una guardería hasta las ocho de la tarde todos los días. (Hombre, 32 años, estudios superiores)

[...]

Sí, que es muy difícil (tener hijos). Porque para tener un hijo necesito tener una estabilidad, una casa, un hogar, es decir, tener algo seguro o tener unas condiciones económicas o un salario razonablemente elevado, y luego tener tiempo para criarlo. Porque si tienes un hijo es una preocupación y tienes que educarle y estar pendiente de él, y si estás trabajando ocho, diez o doce horas al día no vas a estar un poquito por la noche y luego los fines de semana. (Hombre, 27 años estudios superiores)

Como veremos en el apartado siguiente, la actitud de estos jóvenes y su disposición a participar en el trabajo doméstico y en el cuidado de los hijos y las hijas choca, sin embargo, con la organización de los horarios laborales y con las prácticas de gestión del tiempo de las organizaciones en las que trabajan. 


\section{Tiempo de trabajo, maternidad y paternidad}

La excesiva duración de la jornada laboral y la forma en que están organizados los horarios hace difícil que el desempeño de una ocupación resulte compatible con la dedicación a cualquier otra actividad. La visibilidad y la permanencia en el lugar de trabajo más allá del horario establecido son interpretadas frecuentemente por las empresas como señales de implicación y compromiso organizacional, una práctica que es especialmente frecuente en los niveles altos de la escala ocupacional. La mayoría de los y las jóvenes entrevistadas en este estudio señalan estos rasgos como característicos de las organizaciones en las que trabajan:

Pero luego, jes que son unos horarios laborales!, es que es de ocho y media que yo entro por las mañanas, hasta que llego a mi casa. Que muchas veces llego a las nueve y media, las ocho, como pronto las siete, como pronto las siete. Dónde está ahí, o sea no hay guardería que te soporte eso. Y aparte que no hay guarderías, bueno sí echo mano de los abuelos. Pero es que no ves al niño en todo el día, es que no lo ves, no lo ves. Los fines de semana, y los fines de semana a lo mejor estás tan hecha polvo que a lo mejor tampoco puedes dedicarle mucho tiempo, ¿no? Entonces es un tema complicado. (Mujer, 30 años, estudios superiores) [...]

$\mathrm{Y}$ ya no es el horario legal que tenemos, que es de ocho a tres, sino que encima ellos quieren que te quedes hasta las ocho o las nueve de la noche y encima sin pagarte las horas extra. (Hombre, 32 años, estudios medios)

El hecho de que la disponibilidad y la dedicación exclusiva sean los principales criterios para evaluar el compromiso con la empresa no solo afecta la organización de los horarios y la duración de la jornada diaria, sino que también influye en la posibilidad que las personas tienen de gestionar el tiempo dedicado al trabajo durante su carrera laboral. Las exigencias de disponibilidad y permanencia de las organizaciones laborales dificultan que aquellas personas implicadas en el desarrollo de una carrera profesional puedan abandonar temporalmente el empleo para atender otras responsabilidades, como, por ejemplo, el cuidado de los hijos y las hijas. Esta forma de entender la motivación y la implicación representa un obstáculo para las mujeres que desean compaginar su carrera laboral con la maternidad, al tiempo que se convierte en un freno para la mayor participación del hombre en el cuidado de los hijos. La posibilidad legal de que el padre abandone temporalmente el mercado de trabajo para participar en las tareas de crianza choca frontalmente con esta definición del compromiso con la empresa:

En la empresa privada olvídate de un año de excedencia. Los mesecitos que te toquen de baja y gracias, y bueno y estás ahí. Porque es que no eres competente con tus compañeros hombres. Si te vas, te quedas desfasada. Y de hecho yo tengo compañeras que han tenido niños, porque mi empresa en ese aspecto yo pienso que sí que es comprensiva, pero bueno que no se han cogido las excedencias. Se han cogido las bajas exactamente y han vuelto otra vez al trabajo, y no en el mismo puesto que tenían y contentas porque les han mantenido las condiciones. (Mujer, 30 años, estudios superiores)

[...]

Hablando con mi pareja hemos pensado en compaginar el tema del cuidado del niño. En cuanto terminara ella su permiso pues yo pediría un permiso a mi empresa y continuar esos meses hasta que pudiéramos. También hemos pensado que si nos hiciera falta pedir una excedencia o pedir tiempo a la empresa para seguir cuidando al niño (.....) pero cuando quieres acceder a un puesto de dirección lo que miran es que hayas tenido una evolución continua en tu carrera, aumentando de puesto de trabajo y de responsabilidad. Claro, tener un espacio de tiempo para cuidar un niño, cuidar un familiar...., pues no lo ven nada bien e incluso lo ven como una debilidad a la hora de integrarte en el proyecto de la empresa. (Hombre, 27 años, estudios superiores)

La cultura organizacional que predomina en la actualidad, en la que se valora sobre todo el tiempo que la persona permanece en su puesto, unida a la persistencia de una visión estereotipada de la maternidad y la paternidad sitúa a las mujeres en una posición de clara desventaja frente a los varones. Mientras que en el caso de estos la dedicación exclusiva al empleo es compatible con la definición tradicional de la paternidad - cuya función principal es el sustento económico-, en el caso de las mujeres los requerimientos temporales de la actividad laboral entran en conflicto con las demandas del rol maternal. El tiempo, uno de los recursos más valorados por las organizaciones empresariales, se percibe como típicamente 
masculino, lo que se deriva del hecho de que los hombres siguen exentos de la responsabilidad del trabajo doméstico. En contraste, la falta de tiempo que se considera una consecuencia inevitable del rol maternal- se percibe como la principal limitación de las mujeres a la hora de desarrollar una carrera laboral:

Hay muchas mujeres. Pero yo lo que estoy notando, por lo menos en la región en la que yo estoy, que llegan a un nivel en el cual ya pues se estancan un poco. De hecho, en la región donde estoy yo no hay ninguna directora. $\mathrm{O}$ sea, que ya es significativo. $\mathrm{Y}$ es un poco pensando... Pues porque normalmente todas las mujeres que hay son jóvenes y entonces ven que en cualquier momento van a tener hijos y yo creo que es por eso, aunque no es algo que se les diga oficialmente ni mucho menos. Pero creo que influye mucho a la hora de que no las hagan directoras, por el tema de que luego están embarazadas, pues sería para la empresa un problema, yo creo. Y no veo que pongan ninguna solución, y no ya en mi Banco, lo veo en todo el sector. En otros bancos, incluso peor. Incluso pues desde despedir a mujeres que han tenido hijos y han tenido que sacrificar un poco el horario que tenían para atender a los hijos. (Hombre, 32 años, estudios medios)

$[\ldots]$

Por norma general, sí que prefieren que no tengas hijos, y si no tienes pareja, mejor, que así ya no te vas a casar y no te vas a meter en tonterías. De hecho, a mí me preguntaron que si tenía idea de casarme y tal, en la entrevista. Y en más de una entrevista me he encontrado con eso de que si quieres tener niños (...) Porque quieras o no, son cuatro meses que luego tienen que buscar a alguien, más los días que vas a faltar porque te tienes que hacer las pruebas, tus historias... Te casas, tienes una casa e igual, estás como que la mujer tiene que pringar más que el hombre. Entonces, todo eso lo tienen en cuenta, yo creo. Pero yo no me siento discriminada. (Mujer, 27 años, estudios medios)

[...]

Porque es que yo veo a mis compañeras que, las que tienen niños ¡uf! El otro día una compañera estaba ya en la oficina y gritando, "es que no veo a mi hija, me tengo que ir, porque es que me tengo que ir". Y le habían puesto una reunión a las seis de la tarde, que es la hora de salida y "no voy a la reunión y es que no voy porque es que tengo a mi hija mala y es que no la he visto en todo el día. $\mathrm{Y}$ no puedo ir. ¿Y es que por qué me pones la reunión a las seis, por qué no me la pones a las cuatro?". Y claro eso es un hándicap enorme, porque claro, todos tus compañeros varones están ahí y tú no vas a estar en la reunión importante con el cliente. Porque el cliente te ha dicho que puede a las seis y no a las cuatro y es así, el cliente manda. (Mujer, 30 años, estudios superiores)

En definitiva, la organización actual de los tiempos laborales dificulta considerablemente la conciliación entre la vida laboral y la vida familiar no solo para las mujeres, sino también para los hombres. Sin embargo, mientras que en el caso de los varones las reglas del mercado no entran en contradicción con el desempeño de sus roles tradicionales, en el caso de las mujeres suponen un claro conflicto que desencadena un alto grado de estrés y con frecuencia desemboca en el abandono del mercado laboral.

Muy mal por lo de la maternidad, sobre todo. Porque lo estoy viendo ahora con unas amigas embarazadas, compañeras... Pues lo veo mal porque te dan muy poco tiempo, luego tienen mucho problema para encontrar guarderías que sean pues del Estado, las de pago son muy caras y tienen muchas pegas, tienen que buscar a alguien que les cuesta muy caro y muchas de ellas dejan de trabajar porque dicen que no les compensa pagar una persona que es casi su sueldo y encima están perdiendo el ver al niño. (Mujer, 26 años, estudios medios)

$[\ldots]$

Yo ahí fue impotencia. Porque es que ya está claro que yo he traído niños al mundo porque de alguna manera quiero disfrutar de ellos. Y entonces entiendo que los primeros años de su vida son importantísimos. Y es que es así, necesitan a la madre o al padre. Y el hecho de que estuviera ocho horas fuera de su lado o más. Porque en ese momento a lo mejor no eran ocho horas reales, ¿no?, porque había estado por la mañana con él, es un horario que tenía flexible. Pero a esas horas por la noche que ya se supone que le tienes que, que ellos tienen un horario y una rutina diaria cuando son bebés que no es conveniente que lo rompas. Entonces cuando no puedes estar para la cena, para el baño pues es duro, es duro. La verdad a mí es que hubo días como te cuento de llegar llorando. Porque digo a mí no me compensa esto. El hecho de estar ganando un sueldo. Y ya cuando tuve al segundo hijo, pues lo dejé, ya no podía compaginarlo. (Mujer, 34 años, formación profesional) 


\section{Discusión y conclusiones}

En este artículo hemos analizado la percepción que los y las jóvenes tienen de la maternidad y la paternidad, y la forma en que dicha percepción podría influir en la trayectoria laboral de hombres y mujeres. El desigual reparto del trabajo entre hombres y mujeres - tanto remunerado como no remunerado- está asociado, entre otros factores, a creencias estereotipadas sobre la existencia de una diferenciación marcada entre las características psicológicas masculinas y femeninas. Este tipo de creencias adquiere una especial centralidad cuando se valora el papel de hombres y mujeres en la crianza de los hijos y las hijas.

El discurso sobre la maternidad se caracteriza por una marcada esencialización de las características psicológicas femeninas. Las expectativas asociadas al rol maternal están sustentadas en la creencia según la cual los procesos biológicos que rodean al embarazo y al parto dotan a las mujeres de una serie de rasgos psicológicos que las predispone de forma natural para el cuidado y la crianza. Muchas mujeres jóvenes entienden aún la maternidad en términos de disponibilidad absoluta y dedicación exclusiva, lo que las lleva a contraponer el rol maternal con el rol laboral, como si ambos tuvieran que entrar necesariamente en conflicto. El rechazo de un modelo de familia sustentado en la división tradicional de los roles de género coexiste con una fuerte identificación con el rol maternal tradicional. Como lo señala Beck (1998, p. 139),

$\mathrm{Al}$ interés de las mujeres por asegurar su autonomía económica y por llevar a cabo una actividad profesional individualizadora se le sigue contraponiendo el interés por la relación de pareja y por la maternidad, también y precisamente en el caso de aquellas mujeres que saben lo que significa esto para sus oportunidades profesionales y para su dependencia económica respecto del marido. La oscilación entre la vida propia y la existencia para otros con una conciencia nueva muestra la indecisión del proceso de individualización de las mujeres.

La percepción estereotipada de la maternidad no incluye solo la creencia de que las mujeres poseen la capacidad innata para detectar las necesidades emocionales del hijo, sino que también lleva implícita la idea de que la poseen en exclusiva. A la imagen de la madre preocupada y en permanente estado de alerta se contrapone con frecuencia la del padre tranquilo y un tanto despreocupado. Frente a la intuición y la capacidad de empatía de la mujer se enfatiza la torpeza de los varones para desenvolverse en la esfera afectiva. Estos estereotipos negativos que las mujeres mantienen sobre los varones - observados también en otros estudios (Eagly y Mladinic, 1989; 1994; Glick y Fiske, 2001b; 2001c)—, pueden entenderse como una reacción ante la discriminación, la cual cumple la función de reforzar la identidad y la autoestima. Como sugieren Glick y Fiske (2001a), podríamos considerarlos como una diferenciación de género compensatoria que permite a las mujeres diferenciarse positivamente de los hombres en aquellos ámbitos en los que se sienten más seguras o en los que tienen más poder, como puede ser el ámbito doméstico. Se trataría de una forma de prejuicio benevolente, una especie de maternalismo por el que la mujer aparece como más competente y dominante, pero que a la larga perpetúa su posición subordinada.

Los estereotipos de género que se construyen alrededor de la maternidad son uno de los factores ideológicos que mantienen la discriminación de las mujeres dentro del mercado laboral. La incompatibilidad entre el ideal social de la buena madre y la organización de los tiempos de la actividad laboral es uno de los mecanismos que explica las desventajas de las mujeres en el mercado laboral. En el caso de los y las jóvenes que desempeñan trabajos poco cualificados, la prolongación de las jornadas laborales, junto con la escasa cuantía de los salarios, hace difícil conciliar el trabajo con el cuidado de los hijos. Dado que este se percibe todavía como una responsabilidad de la madre, son las mujeres las que abandonan el mercado laboral cuando el cuidado de los hijos no puede ser delegado en personas remuneradas. En el caso de los jóvenes más cualificados, uno de los principales mecanismos de exclusión laboral de las mujeres es la incompatibilidad entre las expectativas del rol maternal y la definición del compromiso organizacional que caracteriza a la mayoría de las empresas. Las organizaciones empresariales siguen asumiendo que las personas que se integran en el mercado laboral no tienen más responsabilidad que el empleo, y exigen a sus trabajadores disponibilidad absoluta y dedicación exclusiva. La cultura organizacional predominante premia la visibilidad y la permanencia como señales de implicación en el trabajo, por lo que la disponibilidad de tiempo se define como uno de los rasgos más valorados por parte de las empresas, sobre todo en el caso del personal directivo. Esto coloca a las mujeres en una posición de clara desventaja en el mercado 
laboral, ya que el tiempo se percibe como un recurso típicamente masculino, lo cual se basa en el predominio de una imagen estereotipada sobre la maternidad y la paternidad por la que los hombres estarían exentos del trabajo doméstico. En un momento en el que las mujeres jóvenes se han equiparado a los varones en términos de capacidad, formación y experiencia, la forma en la que la disponibilidad de tiempo es entendida por las organizaciones laborales se ha convertido en un importante factor diferenciador que dificulta la promoción de las mujeres y las excluye de los sectores ocupacionales de mayor estatus (Wynn, 2017).

Esta organización de los tiempos laborales dificulta también la implicación de los varones en el trabajo no remunerado. Aunque lentamente, la participación del padre en el cuidado de los hijos está aumentando, algo especialmente perceptible en los varones con mayor nivel educativo y en aquellos cuya pareja está implicada en el desarrollo de una carrera profesional (Meil y Rogero, 2014; Tobío, 2012). En el discurso de algunos de los jóvenes que participaron en este estudio se hace patente este cambio en la definición de la paternidad, la cual ya no se entiende únicamente en términos de sustento económico de los hijos. Al igual que las mujeres, algunos varones reflexionan sobre su disponibilidad de tiempo para estar con los hijos, y sobre el obstáculo que una mayor presencia en el ámbito familiar puede suponer para el desarrollo de su carrera.

Aunque cada vez son más las mujeres que combinan las responsabilidades familiares con jornadas prolongadas, esto tiene un coste psicológico alto, desencadena altos niveles de estrés y da lugar a fuertes sentimientos de culpa por no atender de forma adecuada a la familia. Por esto, la forma en la que están organizados los tiempos laborales ha sido considerada como un freno que bloquea la promoción de las mujeres a puestos de mayor responsabilidad (Rutherford, 2001). Esta forma de entender el compromiso con el trabajo es una de las contradicciones del actual discurso de la igualdad, ya que al mismo tiempo que en la mayoría de las empresas se adoptan políticas de igualdad y conciliación, se mantienen unos horarios que perpetúan la tradicional división de roles.

\section{Referencias}

Allport, G. W. (1954). The Nature of Prejudice. Nueva York: Doubleday Anchor.

Beck, U. (1998). La sociedad del riesgo: hacia una nueva modernidad. Barcelona: Paidós.
Bobel, C. (2002). The Paradox of Natural Motherhood. Filadelfia: Temple University Press.

Bravo, P. M., Figueras-Maz, M., y Gómez-Puertas, L. (2014). El ideal de madre en el siglo xxI. La representación de la maternidad en las revistas de familia/The ideal mother in the xxI century. The representation of motherhood in family magazines. Estudios sobre el Mensaje Periodístico, 20(1), 487-504.

Broverman, I. K., Vogel, S. R., Broverman, D. M., Clarkson, F. E. y Rosenkrantz, P. S. (1972). Sex-Role Stereotypes: A Current Appraisal. Journal of Social Issues, 28(2), 59-78.

Carrasquer, P. (2009). El empleo femenino en España y Europa: cambios y continuidades. En J. Astelarra (Coord.), Género y empleo (pp. 15-23). Madrid: Fundación Carolina.

Castillo-Mayén, R. y Montes-Berges, B. (2014). Analysis of Current Gender Stereotypes. Anales de Psicología, 30(3), 1044-1060.

Centro de Investigaciones Sociológicas-CIs. (2004). Estudio 2578. Actitudes y opiniones sobre la familia. Recuperado de http://www.cis.es/cis/opencm/ES/ 1_encuestas/estudios/ver.jsp?estudio $=4556$

Crosby, F., Williams, J. y Biernat, P. (Eds.). 2004. The Maternal Wall. Research and Policy Perspectives on Discrimination Against Mother (Special Issue). Journal of Social Issues, 60(4), 675-682.

Cuddy, A., Fiske, S. T. y Glick, P. (2004). When Professionals Become Mothers, Warmth Doesn't Cut the Ice. Journal of Social Issues, 60(4), 701-718.

Cundiff, J. L. y Vescio, T. K. (2016). Gender Stereotypes Influence How People Explain Gender Disparities in the Workplace. Sex Roles, 75(3-4), 126-138.

Deaux, K. y Lewis, L. L. (1984). Structure of gender stereotypes: interrelationships among components and gender label. Journal of Personality and Social Psychology. 46, 991-1004.

Domínguez-Folgueras, M. (2010). ¿Cada vez más igualitarios? Los valores de género de la juventud y su aplicación en la práctica. Revista de Estudios de Juventud, 90, 103-122.

Durán, M. A. (2000). La contribución del trabajo no remunerado a la economía española. Alternativas metodológicas. Madrid: Instituto de la Mujer. Ministerio de Asuntos Sociales y Trabajo.

Durán, M. A. (2006). Sociopsicología del trabajo no remunerado. En A. Garrido (coord.), Sociopsicología del Trabajo (pp. 133-176). Barcelona: Uoc. 
Durán, M. A. (2005). El trabajo no remunerado y las familias. Aecqualitas. Revista Jurídica de Igualdad de Oportunidades entre Mujeres y Hombres, 17, 47-59.

Durán, M. A. y Rogero, J. (2005). Nuevas parejas para viejas desigualdades. Revista de Estudios de Juventud, 1, 25-37.

Eagly, A. H. (1987). Sex Differences in Social Behavior: A Social-Role Interpretation. Hillsdale, NJ: Lawrence Erlbaum Associates.

Eagly, A. H. y Mladinic, A. (1989). Gender Stereotypes and Attitudes Toward Women and Men. Personality and Social Psychology Bulletin, 15, 543-558.

Eagly, A. H. y Mladinic, A. (1994). Are People Prejudiced Against Women? Some Answers from Research on Attitudes, Gender Stereotypes, and Judgments of Competence. European Review of Social Psychology, 5, 1-35.

Eagly, A. H. y Steffen, V. J. (1984). Gender Stereotypes Stem from the Distribution of Women and men into Social Roles. Journal of Personality and Social Psychology, 46, 735-754.

Fiske, S. (1993). Controlling Other People. The Impact of Power on Stereotyping. American Psychologist, 48(6), 621-628.

Fiske, S., Cuddy, A., Glick, P. y Xu, J. (2002). A Model of (often mixed) Stereotype Content: Competence and Warmth. Respectively Follow from Perceived Status and Competition. Journal of Personality and Social Psychology, 82(6), 878-902. doi: 10.1037//0022-3514.82.6.878

Flaquer, L. y Escobedo, A. (2014). Licencias parentales y política social de la paternidad en España. Cuadernos de Relaciones Laborales, 32(1), 69-99.

García-Román, J. (2012). ¿Son las parejas españolas menos igualitarias que las europeas? Diferencias en el tiempo empleado en trabajo no remunerado en España, Italia, Alemania, Francia y Reino Unido. Documents d' analisi geográfica, 58, 397-416.

Glick, P. y Fiske, S. (1996). The Ambivalent Sexism Inventory: Differentiating Hostile and Benevolent Sexism. Journal of Personality and Social Psychology, 70(3), 491-512.

Glick, P. y Fiske, S. T. (2001a). An Ambivalent Alliance: Hostile and Benevolent Sexism as Complementary Justifications for Gender Inequality. American Psychologist, 56, 109-118.

Glick, P. y Fiske, S. T. (2001b). Ambivalent Sexism. En M. P. Zanna (Ed.), Advances in Experimental Social Psychology (Vol. 33, pp. 115-188). San Diego, CA: Academic Press.
Glick, P. y Fiske, S. T. (2001c). Ambivalent Stereotypes as Legitimizing Ideologies: Differentiating Paternalistic and Envious Prejudice. En J. T. Jost (Ed.), TThe Psychology of Legitimacy: Emerging Stereotypes of Working Moms (pp. 278-306). Nueva York: Cambridge University Press.

Hays, S. (1996). The Cultural Contradictions of Motherhood. New Haven, ст: Yale University Press.

Heilman, M. E. (2012). Gender Stereotypes and Workplace Bias. Research in Organizational Behavior, 32, 113-135. doi: 10.1016/j.riob.2012.11.003

Heilman, M. E. y Eagly, A. H. (2008). Gender Stereotypes Are Alive, Well, and Busy Producing Workplace Discrimination. Industrial and Organizational Psychology, 1, 393-398.

Heilman, M. E. y Okimoto, V. (2008). Motherhood: A Potential Source of Bias in Employment Decisions. Journal of Applied Psychology. 93(1), 189-198.

Hoffman, C. y Hurst, N. (1990). Gender Stereotypes: Perception or Rationalization? Journal of Personality and Social Psychology, 58, 197-208.

Instituto Nacional de Estadística. [INE]. (1976). Encuesta de población activa. Madrid: Instituto Nacional de Estadística. Recuperado de http://www.ine.es

Instituto Nacional de Estadística. [INE]. (2017). Encuesta de población activa. Madrid: Instituto Nacional de Estadística. Recuperado de http://www.ine.es

Jackman, M. R. (1994). The Velvet Glove: Paternalism and Conflict in Gender, Class, and Race Relations. Berkeley: University of California Press.

Jost, J. T. y Banaji, M. R. (1994). The Role of Stereotyping in System-Justification and the Production of False Consciousness. British Journal of Social Psychology. 33, 1-27.

Jost, J. T. y Kay, A. C. (2005). Exposure to Benevolent Sexism and Complementary Gender Stereotypes: Consequences for Specific and Diffuse Forms of System Justification. Journal of Personality and Social Psychology, 88(3), 498-509.

Koening, A. M. y Eagly, A. H. (2014). Evidence for The Social Role Theory of Stereotype Content: Observations of Groups' Roles Shape Stereotypes. Journal of Personality and Social Psychology, 107, 371-392.

Meil, G. y Rogero, J. (2014). Abuelas, abuelos y padres varones en el cuidado de la infancia. Cuadernos de Relaciones Laborales, 32(1), 49-67.

Oakley, A. (1979). Becoming a Mother. Oxford, RU: Martin Robertson. 
Osborne, R. (1987). Simmel y la cultura femenina. Las múltiples lecturas de unos viejos textos. Reis. Revista Española de Investigaciones Sociológicas. 40, 97-112.

Park, B., Banchefsky, S. y Reynolds, E. B. (2015). Psychological Essentialism, Gender, and Parenthood: Physical Transformation Leads to Heightened Essentialist Conceptions. Journal of Personality and Social Psychology, 109(6), 949-967.

Pastor, I. (2009). Las mujeres jóvenes en el mercado de trabajo: entre los datos y los discursos. En J. Astelarra (Coord.), Género y empleo (pp. 79-86). Madrid: Fundación Carolina.

Paterna, C., Martínez, C., Rosa, A. y Yago, C. (2001). De la comparación al compromiso. Un análisis de la identidad social femenina. Revista de Psicología Social Aplicada, 11(2), 5-22.

Ridgeway, C., y Correll, S. J. (2004). Motherhood as a Status Characteristic. Journal of Social Issues, 60(4), 683-700.

Rodríguez-Menéndez, M. C., y Fernández-García, C. M. (2010). Empleo y maternidad: el discurso femenino sobre las dificultades para conciliar familia y trabajo. Cuaderno de Relaciones Laborales, 28(2), 257-275.

Rodríguez, M.C., Peña, J. V. y Torío, S. (2009). La experiencia de la maternidad y la paternidad: análisis del discurso de las creencias sobre la crianza y el cuidado infantil. Infancia y aprendizaje, 32(1), 81-96.

Rosenkrantz, P. S., Vogel, S. R., Bee, H., Broverman, I. K., y Broverman, D. M. (1968). Sex Role Stereotypes and Self-Concepts in College Students. Journal of Consulting and Clinical Psychology, 32, 287-295.

Rutherford, S. (2001). Organizational Cultures, Women Managers and Exclusion. Women in Management Review, 16(8), 371-382. doi: https://doi.org/ 10.1108/ EUM0000000006289
Simmel, G. (1938). Cultura femenina y otros ensayos. México: Espasa Calpe Mexicana.

Spence, J. T., Helmreich, R. L., y Stapp, J. (1975). Ratings of Self and Peers on Sex Role Attributes and their Relation to Self-Esteem and Conceptions of Masculinity and Feminity. Journal of Personality and Social Psychology, 32(1), 29-39.

Sullivan, C. (2015). 'Bad Mum Guilt': the representation of 'work-life balance' in UK women's magazines. Community, Work \& Family, 18(3), 284-298.

Tajfel, H. (1981). Human Groups and Social Categories. Cambridge, Inglaterra: Cambridge University Press.

Tobío, C. (2012). Cuidado e identidad de género. De las madres que trabajan a los hombres que cuidan. Revista Internacional de Sociología, 70(2), 399-422. doi: 10.3989/ris.2010.08.26

Torns, T. y Recio, C. (2012). Las desigualdades de género en el mercado de trabajo: entre la continuidad y la transformación. Revista de Economía Crítica, 14, 178-202.

Wall, G. (2013). 'Putting family first': shifting discourses of motherhood and childhood in representations of mothers' employment and child care. Women's Studies International Forum, 40, 162-171. doi: 10.1016/j. wsif.2013.07.006

Williams, J. E. y Best, D. L. (1982). Measuring Sex Stereotypes: A Thirty-Nation Study. Beverly Hills, ca: Sage.

Wynn, A. (2017). Gender, parenthood, and perceived chances of promotion. Sociological Perspectives, 60(4), 645-664. 